\title{
Searching with an Autonomous Robot
}

\author{
Sándor P. Fekete \\ Department of Mathematical \\ Optimization \\ TU Braunschweig \\ D-38106 Braunschweig \\ Germany \\ s.fekete@tu-bs.de
}

\author{
Rolf Klein \\ Institute of Computer Science \\ University of Bonn \\ D-53117 Bonn \\ Germany \\ rolf.klein@uni-bonn.de
}

\author{
Andreas Nüchter \\ Fraunhofer Institute for \\ Autonomous Intelligent \\ Systems \\ D-53754 Sankt Augustin \\ Germany \\ andreas@nuechti.de
}

\section{Categories and Subject Descriptors}

I.2.9 [ Computing Methodologies ]: Artificial Intelligence - Robotics

\section{General Terms}

Autonomous vehicles

\section{Keywords}

Searching, visibility problems, watchman problems, online problems, competitive ratio, three-dimensional laser scanning, autonomous mobile robots, Kurt3D.

\section{INTRODUCTION}

We demonstrate how one of the classical areas of computational geometry has reached practical application, which in turn gives rise to new, fascinating geometric problems. In particular, we discuss the problem of developing a good online strategy for an autonomous mobile robot to locate an object that is hidden behind a corner or door.

Visibility-based problems of surveying, guarding, or searching have a long-standing tradition in the field of computational optimization: Using stationary positions for guarding a region is the well-known art gallery problem. The watchman problem asks for a short tour along which one mobile guard can see the entire region. If the region is unknown in advance, we are faced with the online watchman problem, for which Hoffmann et al. [3] have achieved a constant competitive ratio. In this context, a crucial issue is the question of how to look around a corner: Given a starting position, and a known distance to a corner, how should one move in order to see a hidden object (or the other part of the wall) as quickly as possible? This problem was solved by Icking et al. [4] who showed that an optimal strategy can be characterized by a differential equation that yields a competitive factor of $1.2121 .$. , which is optimal.

Copyright is held by the author/owner.

Dagstuhl-Seminar "Algorithms for Optimization with Incomplete Information Jan 16-21, 2005
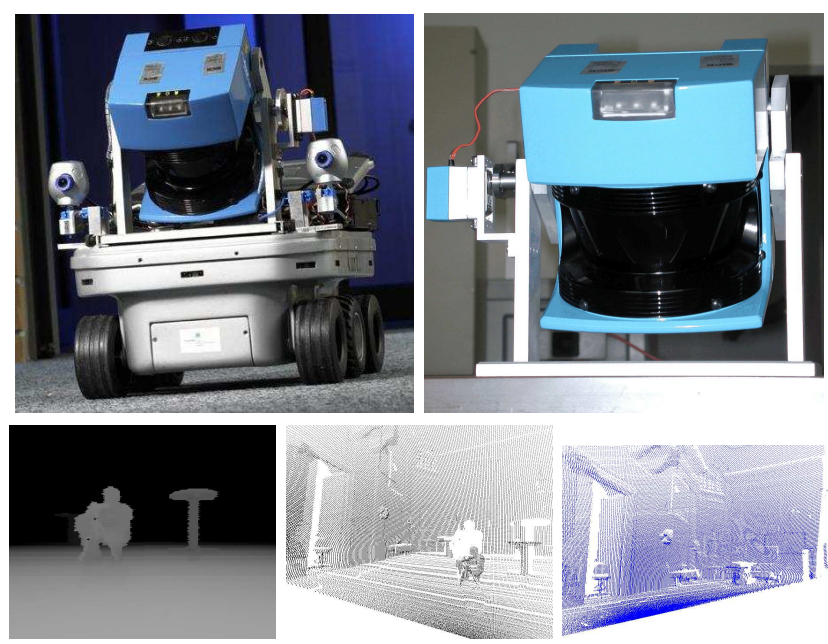

Figure 1: Top left: The autonomous mobile robot Kurt3D equipped with the 3D scanner. Top right: The AIS 3D laser range finder. Its technical basis is a SICK 2D laser range finder (LMS-200). Bottom row, left: A scanned scene as depth image. Middle and right: Scanned scenes as point cloud viewed with a camera orientation towards the door.

From the practical side, our work is motivated by an actual application in robotics: The Fraunhofer Institute for Autonomous Intelligent Systems (AIS) has developed autonomous mobile robots that can survey their environment by virtue of a high-resolution, 3D laser scanner [6]. By merging several 3D scans acquired in a stop, scan, plan, go fashion, the robot Kurt3D builds a virtual 3D environment that allows it to navigate, avoid obstacles, and detect objects [5]. This makes the visibility problems described above quite practical, as actually using good trajectories is now possible and desirable.

However, while human mobile guards are generally assumed to have full vision at all times, our autonomous robot has to stop and take some time for taking a survey of its environment. This makes the objective function (minimize total time to locate an object or explore a region) a sum of travel time and scan time; a somewhat related problem is searching for an object on a line in the presence of turn cost [1], which turns out to be a generalization of the classical linear search 
problem. Somewhat surprisingly, scan cost (however small it may be) causes a crucial difference to the well-studied case without scan cost, even in the limit of infinitesimally small scan times.

\section{ALGORITHMIC APPROACH}

Besides demonstrating the practical state of the art of mobile robots, we describe a good choice for a search strategy. Determining a globally optimal strategy for any given distance the robot may be facing is not only mathematically tricky (requiring the use of numerical methods), but is also of limited practical use: Resulting trajectories can only be reached with limited accuracy. (In the great hall of Schloss Birlinghoven, Kurt's size is still in the order of $2 \%$ of the room diameter.) It should be noted that even in the theoretical paper [3], semi-circles are considered instead of the solution to the differential equation, in order to allow analysis of the resulting trajectories.

Therefore, consider scan points on the semi-circle of diameter $d$ and suppose $c$ was the optimal competitive ratio; it follows from local optimality that for any scan position, the ratio between achieved solution and optimal solution is equal to $c$. In step $i$, we travel a distance of $x_{i}$; denote by $\varphi_{i}$ the corresponding angle on the semi-circle, i.e., $\varphi_{i}=2 \arcsin \left(\frac{x_{i}}{d}\right)$. By the time we get to scan $n$, we have taken a time of $n+\sum_{i=1}^{n} x_{i}$. The worst-case position for the object is infinitesimally behind position $n-1$, implying $n+\sum_{i=1}^{n} x_{i}=c\left(s_{n-1}+1\right)$, where $s_{n-1}$ is the length of a chord to position $n-1$, i.e., $s_{n-1}=d \sin \left(\sum_{i=1}^{n-1} 2 \arcsin \left(\frac{x_{i}}{d}\right)\right)$. Thus,

$$
x_{n}=c\left(1+d \sin \left(\sum_{i=1}^{n-1} 2 \arcsin \left(\frac{x_{i}}{d}\right)\right)\right)-n-\sum_{i=1}^{n-1} x_{i} .
$$

If this sequence reaches the corner, the ratio of $c$ can indeed be achieved. If it collapses prematurely, $c$ is too small. By performing a binary search, the optimal ratio and the necessary step lengths can be computed extremely fast. Moreover, an analysis of the optimal ratio as a function of $d$ shows that a maximum is reached for $d=4.400875 \ldots$ (which is precisely at the threshold between three and four necessary scans), with a competitive ratio of 2.168544 . This is still within about $2 \%$ of the global optimum. More surprisingly, the ratio approaches 2 quite rapidly as $d$ tends to infinity. (For example, $c=2.001525 \ldots$ is optimal for $d=40$.) As there is a matching lower bound of 2 for all strategies and large $d$, our circle strategy is asymptotically optimal; see Figure 2. This does have practical significance for limited physical distances, as it shows that even for arbitrarily small scan times, there is a relatively simple strategy that achieves the optimal ratio of 2 . It also shows that competitive ratios and strategies for arbitrarily small scan times and no scan times differ significantly.

More details are described in the full paper version [2].

\section{THE VIDEO}

The video starts by describing the motivating art gallery (Scene 1) and watchman problems (Scene 2). Following an introduction of online search problems (Scene 3), Scene 4 shows the problem of online search in a polygon. Scene 5 focuses on the issue of how to look around a corner. We describe aspects of a good competitive ratio, and derive how an optimal solution can be described by a differential equation.

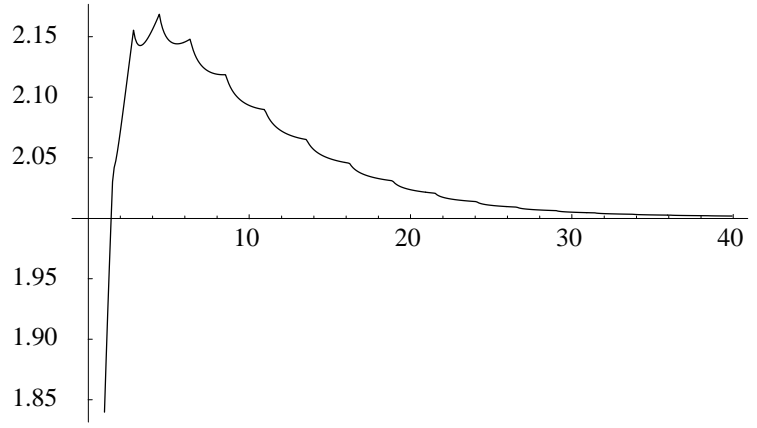

Figure 2: Achieved competitive ratio as a function of $d$.

The next part (Scene 6) introduces Kurt3D and its 3D laser scanner. The virtual drive-through at the end of this scene shows a visualization of the robot's actual vision. This perception is used to locate objects and to plan collision-free trajectories. As shown in Scene 7, the time for performing a scan is in the order of several seconds, but can be adjusted at the expense of resolution.

In the third part, we consider the theory of the resulting online search problem in the presence of scan cost. Starting with a situation in which the time needed to travel to the corner equals the time for performing one scan (Scene 8), we derive a strategy (Scene 9) that is both good (within about $2 \%$ of a global optimum) and relatively easy to evaluate; the latter is crucial for the use in an autonomous robot. Moreover, this strategy is asymptotically optimal (Scene 10).

The fourth part (Scene 11) shows Kurt actually searching the great hall of Schloss Birlinghoven: Starting about 8 meters from a door (1 scan for the right scanner setting), he follows the trajectory developed in the third part; depending on the position of a hidden object (a chair) he may have to perform a second scan from the corner. The second scenario shows a starting distance of 2 scans, resulting in two intermediate scan points.

\section{REFERENCES}

[1] S. P. Fekete, S. Gal, and E. D. Demaine. Online searching with turn cost, 2004. Manuscript (submitted).

[2] S. P. Fekete, R. Klein, and A. Nüchter. Online searching with autonomous robots, 2004. Manuscript (submitted).

[3] F.Hoffmann, C.Icking, R.Klein, and K.Kriegel. The polygon exploration problem. SIAM J. Comp., 31:577-600, 2001.

[4] C. Icking, R. Klein, and L. Ma. How to look around a corner. In Proc. 5th Can. Conf. Comp. Geom., pages 443-448, 1993.

[5] A. Nüchter, H. Surmann, and J. Hertzberg. Automatic Classification of Objects in 3D Laser Range Scans. In Proc. 8th Conf. on Intelligent Autonomous Systems, (to appear) 2004.

[6] H. Surmann, A. Nüchter, and J. Hertzberg. An autonomous mobile robot with a 3D laser range finder for 3D exploration and digitalization of indoor environments. Robotics and Automation, 45:181-198, 2003. 